\title{
Frustrated double ionization of argon atoms in strong laser fields
}

\author{
Seyedreza Larimian, Sonia Erattupuzha, Andrius Baltuška, Markus Kitzler, \\ and Xinhua Xie ${ }^{1}$,
}

Photonics Institute, Technische Universität Wien, A-1040 Vienna, Austria, EU

\begin{abstract}
We report coincidence measurements of frustrated double ionization of argon atoms with a reaction microscope. Experimental results show electron trap- ping process during double ionization has a clear transition from the nonsequential to the sequential regime.
\end{abstract}

\section{Introduction}

The strong field double ionization in atoms and molecules may happen sequentially through directly removing two electrons one after another by the laser field, or nonsequentially, where the first electron releases the second one by re-colliding with the parent ion [1]. After ionization, a fraction of electrons with near zero kinetic energies can be trapped into highlying Rydberg states with binding energies extending from a fraction of an $\mathrm{eV}$ to values of $\mu \mathrm{eV}$ near the ionization threshold [2]. Frustrated double ionization (FDI), which is refereed to such electron trapping process, has been previously investigated for diatomic molecules (including dimers) using Coulomb-explosion imaging technique [3, 4]. In this submission, we report kinematically complete measurements of frustrated double ionization of argon atoms with a reaction microscope.

\section{Experiments and results}
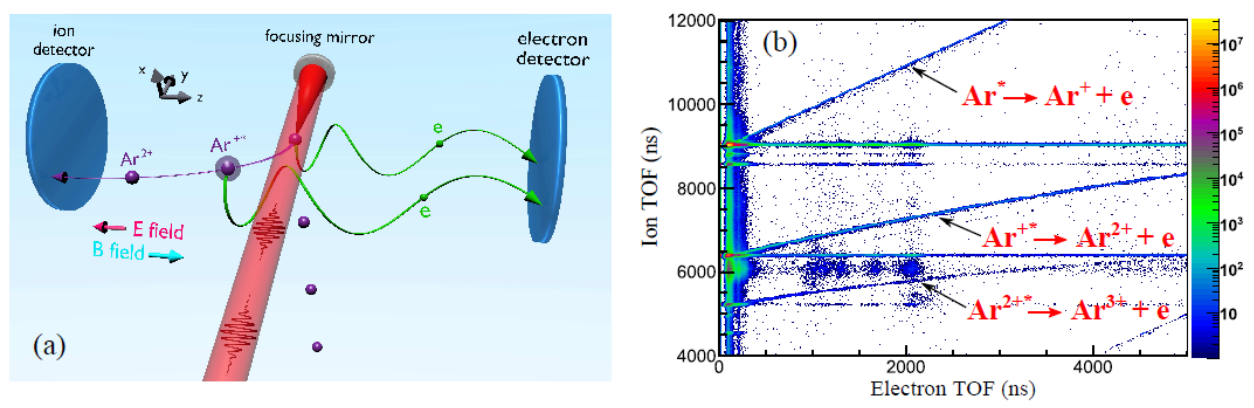

Fig. 1 (a) Schematic view of the experimental setup to achieve the three-body coincidence detection. (b) Measured photo-electron-photo-ion coincidence distribution with the lines representing the

${ }^{1}$ Corresponding author: $\underline{\text { xinhua.xie@tuwien.ac.at }}$ 
correlated signal of electrons and ions from the dc field or BBR ionization.

In the measurements with the reaction microscope [5] we achieved three-body coincidence detection of two electrons and their parent ion produced from the interaction of argon atoms with strong laser pulses. After the strong-field interaction, one (first) electron is removed through strong-field ionization and the other electron is populated to a high-lying Rydberg state. During the flight of the excited ion $\left(\mathrm{Ar}^{+*}\right)$ to the detector, the spectrometer field (with strength of a few $\mathrm{V} / \mathrm{cm}$, which is applied to guide the charged particles to the detectors) or black-body radiation (BBR) further ionizes the highly excited ion and the Rydberg electron is detached. In recent studies, we demonstrated the coincidence detection of Rydberg electrons with ionization induced by the spectrometer field and BBR in the case of single ionization $[6,7]$. The experimental setup and the correlated signals of Rydberg electrons with their parent ions $\left(\mathrm{Ar}^{2+}\right)$, which are represented as a long parabolic curve in the photo-electron-photo-ion coincidence (PEPICO) distribution, are shown in Fig. 1.


Fig. 2 (a) Yields of the measured Rydberg electrons from $\mathrm{Ar}^{*}$ (blue circles) and $\mathrm{Ar}^{+*}$ (red squares) over the emission time. (b) The ratios of trapping probability between the single and double ionization processes as a function of laser peak intensity. The blue dashed line is a fitting curve to guide the eyes.

By normalizing the BBR signal ratio between the Rydberg electrons from $\mathrm{Ar}^{*}$ and $\mathrm{Ar}^{+*}$ respecting the ratio between the signal of strong-field single and double ionization as a function of laser peak intensity, we found that the trapping probability during double ionization is more than one order of magnitude higher than that during single ionization and the ratio between the two probabilities increases with increasing laser intensity, as shown in Fig. 2(b). With the measured time-of-flight and position information of Rydberg electrons and $\mathrm{Ar}^{2+}$, we retrieved the emission time of the Rydberg electron (after the laser interaction) and the momentum vector of $\mathrm{Ar}^{+*}$ produced during strong-field interaction. Since electron momentum distributions contain temporal information of the released electron, we compared electron momentum distributions of frustrated double ionization with strong-field double ionization as a function of laser peak intensity. Electron momentum distributions show that electron trapping dynamics of frustrated double ionization has a clear transition from the non-sequential to sequential double ionization regime. Our results further indicate that in the sequential double ionization regime the trapping process is dominated by the second detached electron because of its smaller quantum diffusion as compared to the first electron. In the nonsequential double ionization regime we find that the trapping probability is strongly suppressed as compared to that in the sequential double ionization regime. We attribute this to the strong correlation between the two electrons which results in momentum distributions offset from zero. 


\section{References}

1. S. Larochelle, A. Talebpour, and S. L. Chin, "Non-sequential multiple ionization of rare gas atoms in a Ti:Sapphire laser field", Journal of Physics B: Atomic, Molecular and Optical Physics 31, 1201 (1998).

2. T. Nubbemeyer, K. Gorling, A. Saenz, U. Eichmann, and W. Sandner, "Strong-Field Tunneling without Ionization", Phys. Rev. Lett. 101, 233001 (2008).

3. B. Manschwetus, T. Nubbemeyer, K. Gorling, G. Steinmeyer, U. Eichmann, H. Rottke, and W. Sandner, "Strong Laser Field Fragmentation of H2: Coulomb Explosion without Double Ionization", Physical Review Letters 102, 113002 (2009).

4. Q. Cheng, X. Xie, Z. Yuan, X. Zhong, Y. Liu, Q. Gong, and C. Wu, "Dissociative Ionization of Argon Dimer by Intense Femtosecond Laser Pulses", The Journal of Physical Chemistry A 121, 3891 (2017).

5. R. Döner, V. Mergel, O. Jagutzki, L. Spielberger, J. Ullrich, R. Moshammer, and H. Schmidt-Bo"cking, "Cold Target Recoil Ion Momentum Spectroscopy: A 'Momentum Microscope' to View Atomic Collision Dynamic", Physics Reports 330, 95 (2000).

6. S. Larimian, S. Erattupuzha, C. Lemell, S. Yoshida, S. Nagele, R. Maurer, A. Baltuška, J. Burgdörfer, M. Kitzler, and X. Xie, "Coincidence spectroscopy of highlying Rydberg states produced in strong laser fields", Phys. Rev. A 94, 033401 (2016).

7. S. Larimian, C. Lemell, V. Stummer, J. Geng, S. Roither, D. Kartashov, L. Zhang, M. Wang, Q. Gong, L. Peng, S. Yoshida, J. Burgdörfer, A. Baltuška, M. Kitzler, and X. Xie, "Localizing high-lying Rydberg wave packets with two-color laser fields", Phys. Rev. A 96, 021403(R) (2017). 